\title{
Abdominal pregnancy misdiagnosed as an intrauterine pregnancy: a cause of failed induction of labour for fetal death
}

\author{
Nkencho Osegi, Olakunle I. Makinde*, Peter O. Eghaghe, Zakaa Zawua, Bright N. Ohaka
}

Department of Obstetrics and Gynecology, Federal Medical Centre, Yenagoa, Bayelsa State, Nigeria

Received: 13 June 2019

Accepted: 09 July 2019

\section{*Correspondence:}

Dr. Olakunle I. Makinde,

E-mail: olakunleife@gmail.com

Copyright: () the author(s), publisher and licensee Medip Academy. This is an open-access article distributed under the terms of the Creative Commons Attribution Non-Commercial License, which permits unrestricted non-commercial use, distribution, and reproduction in any medium, provided the original work is properly cited.

\begin{abstract}
Abdominal pregnancy is a rare form of ectopic pregnancy usually associated with fetal death among other complications, although very rare cases of live births have been reported. There is also a high risk of maternal mortality. A high index of suspicion is required to make a preoperative diagnosis as diagnosis from history, examination and ultrasound is often missed. Misdiagnoses as an intrauterine pregnancy usually occur. This misdiagnosis makes management of patients with an abdominal pregnancy a challenge and may affect treatment outcome. We managed a 35 year old pregnant multipara who was referred to us on account of repeated failed attempts at induction of labour for intrauterine fetal death. Three obstetric ultrasound scans done during the course of patient's management reported an intrauterine dead fetus. We also failed to achieve uterine evacuation. We resorted to carry out a hysterotomy and following laparotomy, we found an abdominal pregnancy. This finding was unexpected by us, however, we delivered the dead fetus and was able to successfully manage the placenta. Discovering an abdominal pregnancy at surgery carried out for a supposed intrauterine pregnancy is usual for many cases of abdominal pregnancy. Clinicians should be aware of the clinical signs and symptoms that raise a suspicion of abdominal pregnancy as prompt preoperative diagnosis of abdominal pregnancy helps to plan and offer early and appropriate intervention. This reduces the incidence of maternal mortality usually due to massive intra-abdominal haemorrhage arising from delayed diagnosis and poor placenta management.
\end{abstract}

Keywords: Abdominal pregnancy, Failed induction of labour, Intrauterine fetal death, Laparotomy, Misoprostol

\section{INTRODUCTION}

Abdominal pregnancy is a rare occurrence associated with high fetal mortality and poses several dilemmas in management. ${ }^{1-3}$ It is often misdiagnosed as an intrauterine pregnancy. ${ }^{4}$ One of such management dilemma is associated with its frequent misdiagnosis as an intrauterine pregnancy. Also in such situation when fetal death occurs, erroneous and futile attempts are often made at inducing labour to evacuate the uterus. Abdominal pregnancy is a rare form of ectopic pregnancy (implantation of a fertilized ovum outside the uterine endometrium) that is implanted in the abdominal cavity. Ectopic pregnancies occur in 1-2\% of pregnancies; about 95\% of ectopic pregnancies are tubal while abdominal pregnancy accounts for just about $1 \% .^{2}$ Abdominal pregnancy is more common in developing countries. ${ }^{1,2}$ It is associated with a high risk of fetal death, fetal growth restriction and congenital malformation. ${ }^{5}$ There is also a significant risk of maternal death from massive haemorrhage especially when not diagnosed early or not properly managed. ${ }^{3}$ It has been reported that the risk of dying from an abdominal pregnancy is 7.7 times greater than the risk of dying from a tubal pregnancy, and 90 
times greater than with a normal intrauterine pregnancy. ${ }^{4,6}$ Maternal mortality rate range from 0.5 $18 \%$; usually due to massive intra-abdominal haemorrhage and infection arising from delayed diagnosis and poor placenta management. , $^{2,3}$ Perinatal mortality rate range from $40-95 \%$; the poor fetal prognosis is largely related to poor vascularization of the placenta, premature placenta aging and associated fetal growth restriction and fetal abnormalities., ${ }^{2,5}$

Abdominal pregnancy can be classified based on pathogenesis into primary and secondary abdominal pregnancy, and based on gestational age at diagnosis into early and advanced (late) abdominal pregnancy. Early and advanced abdominal pregnancies are defined when the gestational age at presentation is equal to or less than 20 weeks and when it is more than 20 weeks respectively. ${ }^{4}$ An advanced abdominal pregnancy is rare as often fetal death or other complications occur earlier in the pregnancy requiring intervention. However, some cases of advanced abdominal pregnancy have been reported and also, cases of the even rarer phenomenon of advanced abdominal pregnancy with a live fetus have been documented. Abdominal pregnancy that implanted directly in intra-abdominal structures excluding the fallopian tubes and the ovaries is primary (primary peritoneal implantation), while it is secondary if implantation follows extrusion of the product of conception from the female reproductive organ. ${ }^{4}$ Mechanism of secondary abdominal pregnancy includes tubal abortion or rupture of a tubal or ovarian pregnancy, rupture or perforation of the uterus and secondary implantation of the trophoblast into the abdominal cavity. Intra-abdominal implantation can be into the large vessels, mesenteries, omentum and vital organs. Availability of blood supply from the site of implantation determines the extent of fetal survival. ${ }^{7}$ Most abdominal pregnancies are secondary as primary abdominal pregnancy is rare. ${ }^{2}$

Several aetiological theories have been described for primary abdominal pregnancy. Criteria for its diagnosis were established by Studdiford in 1942 and include 1. The fallopian tube and ovary should be intact bilaterally, 2. No evidence of a fistulous connection between the uterus and peritoneal cavity, 3 . The pregnancy must be related exclusively to the peritoneal surface and early enough to exclude possibility of secondary implantation after primary nidation in the tube. ${ }^{3,4,7}$ The aetiology of secondary abdominal pregnancy is the same as for the more common tubal ectopic pregnancy; with tubal damage especially from pelvic infection with Chlamydia Trachomatis being most common. This may be responsible for the higher incidence of abdominal pregnancies in developing countries where the burden of pelvic inflammatory disease is high. ${ }^{1,3}$ Other aetiological factors include previous history of ectopic pregnancy, endometrioses, failed intra uterine contraceptive device and high dose progesterone only contraceptive, use of clomiphene citrate, invitro fertilization and embryo transfer, smoking etc.

Diagnosis of abdominal pregnancy is challenging as clinically, there are no symptoms and signs pathognomonic of abdominal pregnancy. Associated symptoms include persistent (nonspecific) abdominal pain most frequently, abdominal pain and vaginal bleeding earlier in pregnancy, painful fetal movement, and gastrointestinal symptoms. Signs include easily palpable fetal parts and abnormal lie. Due to the nonspecific nature of these symptoms and signs, abdominal pregnancy is often missed.

\section{CASE REPORT}

Mrs J.J, a 35 year old G9P3+5(3A) with one previous history of ectopic pregnancy and four previous terminations of pregnancies by manual vacuum aspiration who was referred from a general hospital on account of repeated failed induction of labour for intrauterine fetal death.

Patient had presented to the general hospital on account of her non perception of fetal movement and following her assessment, two obstetric ultrasounds done on the same day in two different facilities diagnosed intrauterine fetal death at 27 weeks and 5 days and 28 weeks and 2 days respectively. Induction of labour was attempted at the referring centre on two occasions with up to 200micrograms of misoprostol and thereafter with intra cervical extra amniotic Foley's catheter with no success. She was receiving care in the index pregnancy in a maternity home and ultrasound scans that were done did not report any abnormality.

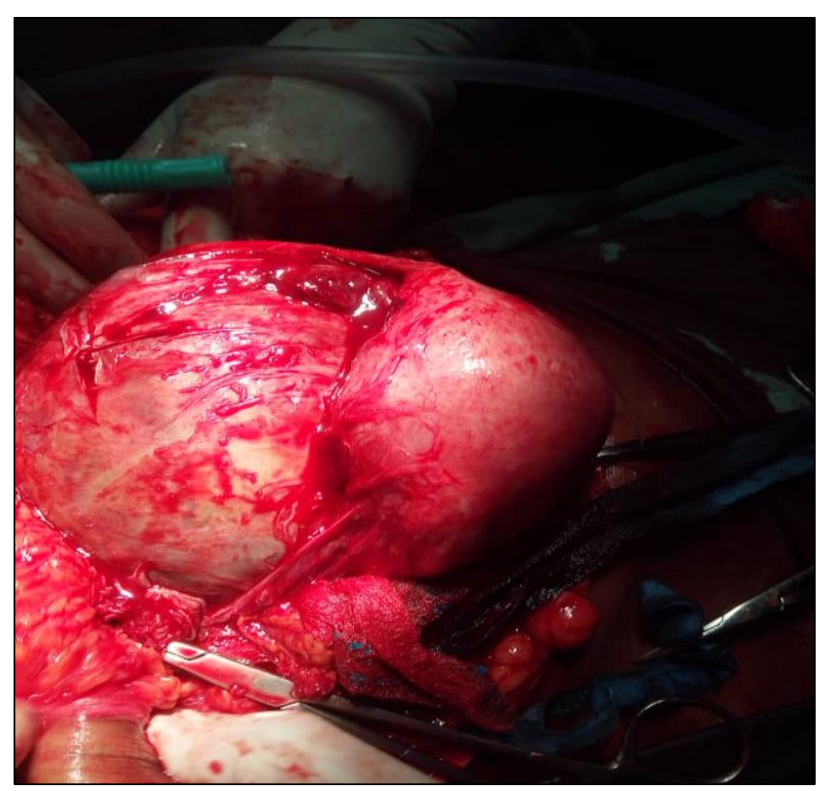

Figure 1: Bulky uterus with the amniotic membrane lying posterior and attached to the uterus. 


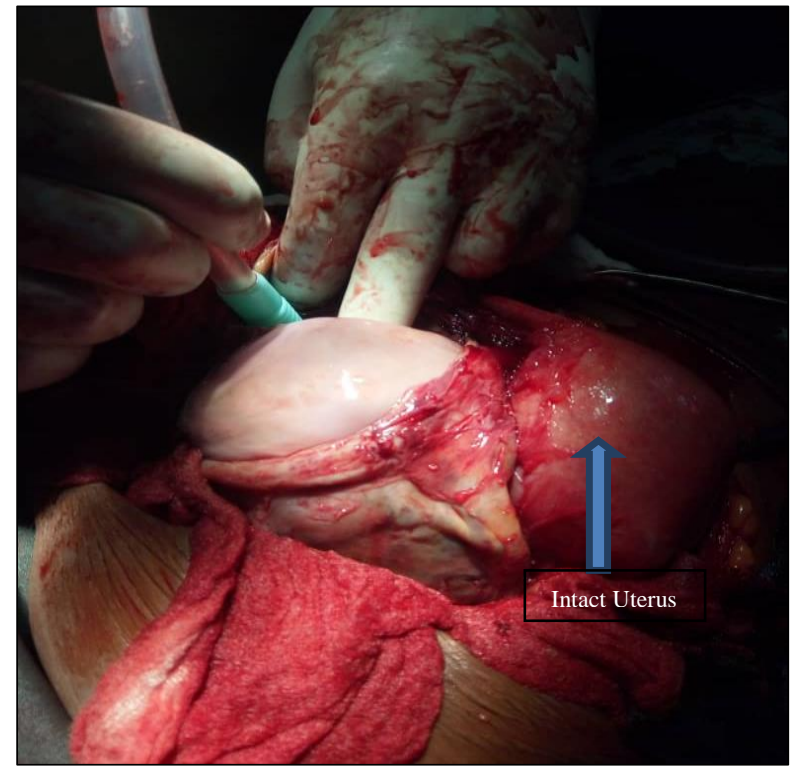

Figure 2: Incised amniotic membrane with fetal breech exposed. Note the adjacent intact uterus.

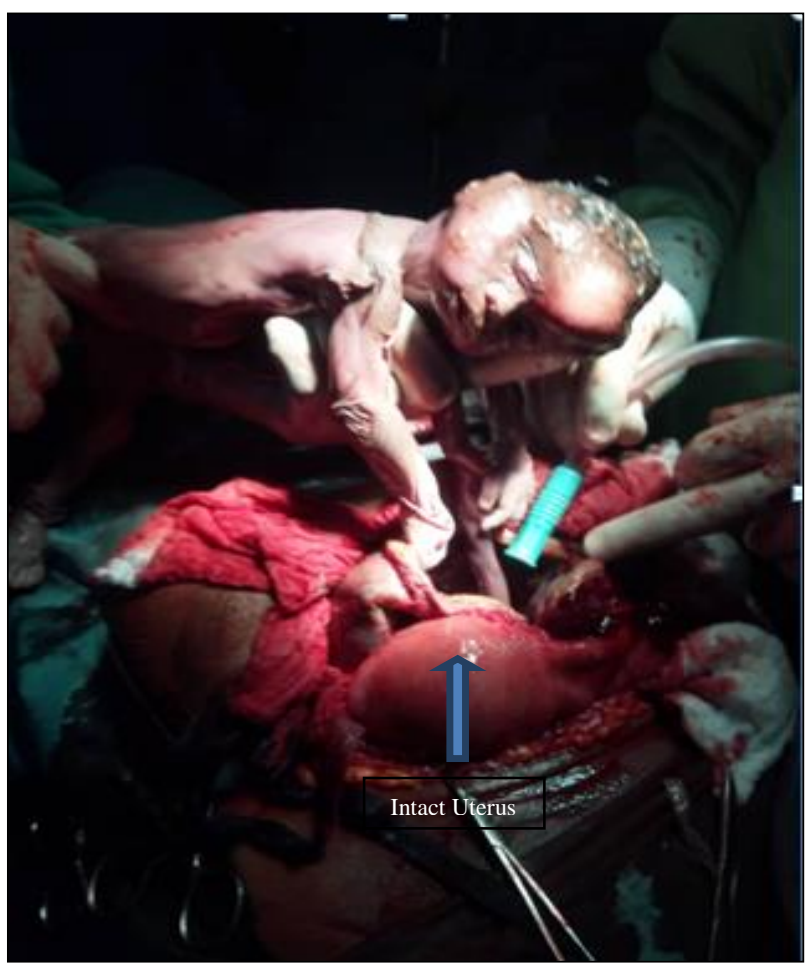

Figure 3: Macerated foetus extracted from within the amniotic membrane. Note the adjacent intact uterus.

Although we found that diagnosis of intrauterine fetal death was made four weeks before, and she failed to present immediately following referral, she had no new complaint at presentation to our facility. Her general condition was stable, and her vital signs were within normal limits except her blood pressure which was 160/100mmHg. Her abdomen was enlarged, symphysiofundal height was $27 \mathrm{~cm}$, a singleton fetus was palpated in longitudinal lie and cephalic presentation, descent was $5 / 5$ th, no palpable uterine contractions, and fetal heart tone was not heard. On vaginal examination, cervix was central, medium in consistency, $1 \mathrm{~cm}$ long, and cervical os was closed. Urinalysis done was negative for protein. A diagnosis of intrauterine fetal death and ? Severe pregnancy induced hypertension was entertained. A repeat obstetric ultrasound done in our facility revealed a single, non active, cephalic presenting intrauterine gestation with overlapping skull bones, surrounding halo and featureless intracranial and intra-abdominal contents. Placenta was left lateral and gestational age was 26 weeks. This confirmed a diagnosis of intrauterine fetal death. Her clotting time, platelet count and full blood count, coagulation profile, serum electrolytes, urea and creatinine, liver function test were normal.

She was offered a repeat attempt at induction of labour and a hysterotomy if induction of labour fails which she accepted. She was placed on oral antihypertensive drugs and on broad spectrum antibiotics. Attempts at induction of labour with vaginal misoprostol at 100micrograms with simultaneous intracervical extra amniotic Foley's catheter failed repeatedly. Intracervical misoprostol was tried when the cervix became dilated to $2 \mathrm{~cm}$ but cervical changes failed to progress further. Oxytocin infusion was also tried despite an unfavourable cervix and unsurprisingly failed. She was subsequently billed for hysterotomy. Preoperative packed cell volume was $31 \%$ and blood grouping and cross matching was done.

Operative findings were a bulky intact but non gravid uterus with an intra-abdominal amniotic membrane measuring about $20 \mathrm{~cm} \times 24 \mathrm{~cm}$ (Figure 1); amniotic membrane was attached to the posterior wall of the uterus, left broad ligament, sigmoid colon, and part of the rectum; left fallopian tube was matted to the amniotic membrane, stretched and dilated, right fallopian tube was buried in adhesion, a macerated male fetus with no gross anomaly, placenta was in most part within the amniotic membrane except at the attachments it had with the left broad ligament and sigmoid mesocolon.

The incision on the skin had to be converted to an inverted $\mathrm{T}$ after a non gravid uterus and an intraabdominal mass was noted. The paracolic gutters and areas around the amniotic membrane were packed with abdominal mops. The macerated fetus was extracted through an incision on the amniotic membrane (Figure 2, 3 ) and umbilical cord cut very close to the placenta. The amniotic membrane was carefully separated from its attachments and was wholly excised with the placenta after we were able to also separate the placenta from its attachments. The sigmoid mesocolon and left broad ligament were repaired and haemostasis was secured. A left salpingectomy was done and a right tubal ligation was also done for completion. The abdomen was lavaged, closed in layers with an abdominal drain left in situ. Blood loss was 2 litres and she was transfused with 4 units of whole blood intraoperatively. 
Her postoperative period was uneventful; postoperative packed cell volume was $33 \%$ (post-transfusion), abdominal drain was removed at 3 rd day postoperative. She was discharged home on the 7 th day postoperative. She was seen at the follow up clinic at 2 weeks postoperative; she had no significant complaint and was stable.

\section{DISCUSSION}

Diagnosis of abdominal pregnancy with symptoms and signs is challenging because some patients are asymptomatic, symptoms and signs are non-specific, and are often missed. Ultrasound is a useful tool for making a diagnosis. Features of early abdominal pregnancy include an empty uterus in the presence of a gestational sac or mass separate from the uterus, adnexa and ovaries. ${ }^{4}$ Ultrasound features of advanced abdominal pregnancy include fetus seen outside the uterine cavity, absence of uterine wall between the bladder and the fetal parts, fetal parts located close to the maternal abdominal wall, placenta located outside the uterus, abnormal looking placenta, and oligohydramnious. ${ }^{6}$ Ultrasound can also access usually associated congenital abnormalities. ${ }^{6}$ Ultrasound diagnosis is however largely dependent on the skill of the operator and gestational age. It is more likely to miss an abdominal pregnancy in the second and third trimesters. $^{7}$ About $50 \%$ of abdominal pregnancies are initially misdiagnosed on ultrasound. ${ }^{4,6,8}$ This statement holds true in our case as our patient did several obstetric ultrasound scans that diagnosed intrauterine fetus. Usually however, diagnosis is made eventually during surgery. Apart from diagnosis made during laparotomy indicated following failed induction of labour for intrauterine fetal death as it was in our case, we found from reviewing literatures that diagnosis have been made during emergency laparotomy for ruptured ectopic pregnancy; during proposed caesarean section indicated by previous diagnosis of prolonged pregnancy and subsequent failed induction of labour, transverse lie at term, placenta previa, bicornuate uterus; or laparotomy for suspicion of uterine rupture. ${ }^{8}$

A high index of suspicion is therefore required for the preoperative diagnosis of an abdominal pregnancy and should be raised when there is persistence of abdominal pain and painful fetal movement, and or abnormal lie, easily palpable fetal parts, prolonged pregnancy, displaced cervix usually non responsive to cervical ripening, abnormally large placenta on ultrasound, repeated failed attempts at uterine evacuation for fetal death. ${ }^{2,3}$ Re-evaluation by ultrasound to rule out abdominal pregnancy preferably by a more experienced hand may clinch the diagnosis. Magnetic resonant imaging (MRI) has a role in diagnosis, it is more accurate and specific and in addition, more relevant for delineating the exact localization of the placenta and fetal parts, and their relationship to vital organs thus helping to determine preoperatively if the placenta can be safely removed or better left in situ. ${ }^{4,6,8}$ Angiograms have a role pre and postoperatively. Preoperatively to locate all sources of vascular supply to the placenta, and postoperatively where the placenta is not removed during laparotomy, to embolize feeding arteries. ${ }^{7} \mathrm{We}$ failed to diagnose abdominal pregnancy preoperatively in our case.

Treatment of abdominal pregnancy is dependent on gestational age at diagnosis, on whether or not fetus is alive, maternal haemodynamic status and on patient's desire. One treatment option is immediate termination of the pregnancy irrespective of gestational age and viability because of the poor fetal prognosis and high risk of massive intra-abdominal haemorrhage from placental disruption and separation anytime during the course of the pregnancy. ${ }^{2,4}$ Surgical termination of early abdominal pregnancy can be achieved by laparotomy and laparoscopy; it however requires surgical expertise and a multidisciplinary team approach in anticipation of possible massive haemorrhage during surgery. ${ }^{4}$ With technological advances, various methods used to control haemorrhage laparoscopically, and increased operator skills; operative laparoscopy rate for early abdominal pregnancy has increased and as published in a case series by Shaw et al, the overall rate was $55 \%$ and it reached $100 \%$ after year $2000 .{ }^{4}$ Medical management also has a role in the treatment of early abdominal pregnancy usually when potential life threatening haemorrhage is anticipated from surgery, such as in early abdominal pregnancy of the liver and spleen. ${ }^{4}$ Agents that have been used include methotrexate (systemic and local), local instillation of potassium chloride, hyperosmolar glucose, prostaglandins, danazol, etoposide and mifepristone. ${ }^{4}$ Patients may however still require surgery after medical management because of haemorrhage and thus should be kept under surveillance. ${ }^{4}$

When abdominal pregnancy is advanced and there is a live fetus, termination of the pregnancy is a complex decision and depends on patient's decision and peculiarities. Conservative management for abdominal pregnancies with a live fetus can be considered when diagnosed after 24 weeks of gestation. ${ }^{2}$ For an advanced abdominal pregnancy diagnosed before 28 weeks gestation, Sapuri and Klufio indicated the possibility of conservative management with the aim of bringing the fetus to viability if the following criteria are met: Fetus is alive; there are no major congenital malformations; there is facility for continuous hospitalization in a wellequipped and well-staffed maternity unit with immediate blood transfusion services; careful monitoring of fetal and maternal wellbeing; and placental is implanted in the lower abdomen away from the liver and spleen. ${ }^{3}$ Sapuri and Klufio also recommended immediate laparotomy and delivery of the fetus once 28 weeks gestation is reached. ${ }^{3}$ However, conservative management can be extended beyond 28 weeks up to 34 weeks to achieve lung maturity especially if patient has been stable. ${ }^{7}$ An advanced abdominal pregnancy diagnosed after 28 weeks gestation with a live fetus requires immediate laparotomy and delivery of the fetus. ${ }^{3}$ An advanced abdominal pregnancy 
with a dead fetus also requires immediate laparotomy and delivery of the fetus. However, the increased risk of disseminated intravascular coagulation and infection should be kept in mind; steps to minimize operative risk must be taken before surgery and surgery should be done as soon as possible. ${ }^{3,4}$

When the fetus has been delivered, management of the placenta becomes the next challenge. Whether or not the placenta should be removed is controversial. There is a high risk of torrential uncontrollable haemorrhage with removal of the placenta irrespective of whether the abdominal pregnancy is early or advanced and whether the fetus is dead or alive. It is wise and acceptable to leave the placenta in situ if removal of the placenta without encountering torrential haemorrhage is not feasible. $^{2}$ It is recommended to ligate the umbilical cord as close as possible to the placenta. ${ }^{3}$ Leaving the placenta in situ is associated with the risks of paralytic ileus, intraabdominal sepsis and abscess collection, secondary haemorrhage, disseminated intravascular coagulation, adhesions, intestinal obstruction, risk of repeat laparotomy and prolonged hospital stay. ${ }^{1,3}$ However, the benefit of avoiding severe haemorrhage associated with attempting to remove the placenta clearly outweighs these risks. Abdominal drain was left in situ more in cases where the placenta was removed. Indications for leaving a drain were peritonitis and oozing of blood at operation site. ${ }^{1}$ The placenta is expected to undergo involution over several weeks to years. Placental involution can be monitored with serial beta-HCG and MRI. ${ }^{3}$ Methotrexate can be used to hasten placental involution, but the side effects of methotrexate which includes a high intra-abdominal infection rate must be balanced against this benefit. ${ }^{1-3}$ In our case, we were able to completely remove the placenta without encountering massive haemorrhage because the bulk of it was within the amniotic membrane and there was just a relatively small area of placenta attachment to the sigmoid mesocolon and left broad ligament. Moreover, the fetal death is likely to be contributory to the ease of placenta removal. Authors left an abdominal drain insitu.

\section{CONCLUSION}

The diagnosis of abdominal pregnancy is often missed. Clinicians need to be aware of the clinical signs and symptoms that should raise a suspicion of an abdominal pregnancy. Medical evacuation of the uterus is ordinarily usually achieved successfully with the use of prostaglandins for induction of labour. When prostaglandins fail repeatedly to induce labour in a case of fetal death, re-evaluation of the patient should be done to rule out an abdominal pregnancy. Early diagnosis, prompt intervention and a right decision on handling the placenta after removal of the foetus are key to patient survival.

\section{Funding: No funding sources Conflict of interest: None declared \\ Ethical approval: Not required}

\section{REFERENCES}

1. Sunday-Adeoye I, Twomey D, Egwuatu EV, Okonta PI. A 30-year review of advanced abdominal pregnancy at the Mater Misericordiae Hospital, Afikpo, south-eastern Nigeria (1976-2006). Arch Gynecol Obstet. 2011;283(1):19-24.

2. Nwobodo EI. Abdominal pregnancy: a case report. Ann Afr Med. 2004;3(4):195-6.

3. Sapuri M, Klufio C. A case of advanced viable extra uterine pregnancy. PNG Med J. 1997;40(1):44-7.

4. Agarwal N, Odejinmi F. Early abdominal ectopic pregnancy: challenges, update, and review of current management. The Obstetrician Gynaecol. 2014;16:193-8.

5. Gabkika BM, Ignonne T, Diara D. Abdominal pregnancy with live fetus at term at the South N'djamena District Hospital: A case study. Sudan JMS. 2015;10(2):77-9.

6. Radhakrishnan K. Intra-abdominal pregnancy. Applied Radiol. 2015;44(5):44-7.

7. Nassali MN, Benti TM, Bandani-Ntsabele M, Musinguzi E. A case report of an asymptomatic late term abdominal pregnancy with a live birth at 41 weeks of gestation. BMC Res Notes. 2016;9(1):31.

8. Deeksha P, Vani R, Jyoti S, Rupinder K, Kamath S. Fetal demise not responding to induction: a case report. Internet J Gynaecol Obstet. 2007;9(1).

Cite this article as: Osegi N, Makinde OI, Eghaghe PO, Zawua Z, Ohaka BN. Abdominal pregnancy misdiagnosed as an intrauterine pregnancy: a cause of failed induction of labour for fetal death. Int $\mathbf{J}$ Reprod Contracept Obstet Gynecol 2019;8:3382-6. 